Review

\title{
Pathophysiologic Implications of Cytokines Secretion during Liver Transplantation Surgery
}

\author{
Wonjung Hwang ${ }^{1}$ and Jaemin Lee ${ }^{2 \bowtie}$ \\ 1. Department of Anesthesiology and Pain Medicine, Seoul St. Mary's Hospital, College of Medicine, The Catholic University of Korea \\ 2. Department of Anesthesiology and Pain Medicine, Uijeongbu St. Mary's Hospital, College of Medicine, The Catholic University of Korea
}

$\triangle$ Corresponding author: Jaemin Lee, M.D., Ph.D. Professor, Department of Anesthesiology and Pain Medicine, Uijeongbu St. Mary's Hospital, College of Medicine, The Catholic University of Korea. 271 Cheonbo-ro, Uijeongbu-si, Gyeonggi-do, Republic of Korea, 11765. E-mail address: jmlee@catholic.ac.kr; Telephone: +8231820 3256; Fax: +82318473449

(C) Ivyspring International Publisher. This is an open access article distributed under the terms of the Creative Commons Attribution (CC BY-NC) license (https://creativecommons.org/licenses/by-nc/4.0/). See http://ivyspring.com/terms for full terms and conditions.

Received: 2018.07.09; Accepted: 2018.10.18; Published: 2018.11.23

\begin{abstract}
We introduce the following issues of the cytokines secretion during liver transplantation surgery in this review article; 1) the aspect of cytokines secretion during liver transplantation surgery, 2) the evidences of association of cytokines concentration with post-transplantation graft survival, 3) a variety of factors that may influence the secretion of cytokines during liver transplantation, 4) pro-inflammatory and anti-inflammatory cytokine balance during the surgery, and 5) the issues of $T$ helper 1 and $T$ helper 2, and $T$ helper 17 and regulatory $T$ cell signature cytokines secretion and their ratio during liver transplantation surgery. Primary failure of the liver is associated with the secondary dysfunction of virtually all other organ systems, including the cardiovascular, pulmonary, renal, coagulation and central nervous systems. In addition, liver transplantation surgery itself is a major surgical procedure with accompanying life-threatening hemorrhage, massive transfusion, clamping and unclamping of great vessels and resulting ischemia-reperfusion injury and cardiovascular instability. Both the underlying liver failure and the surgical events act as stressors and promote the secretion of various cytokines. So it is clinically important to understand above issues regarding the cytokines secretion during liver transplantation surgery. As cytokines secretion has clear relationship with post-transplantation clinical outcomes, future study directions for artificially manipulating cytokines secretion is also suggested for enhancing outcomes of the patients.
\end{abstract}

Key words: cytokine, interleukins, pro-inflammatory, anti-inflammatory, $\mathrm{T}$ lymphocytes, helper-inducer, Th1-Th2 balance, Th17-Treg balance, liver transplantation

\section{Introduction}

Not only underlying diseases that require liver transplantation surgery, but the liver transplantation surgery itself also serves as a significant stressor to the human body. It accompanies life-threatening hemorrhage, massive transfusion, shifts in body fluids, ischemia-reperfusion injury, changes in the utilization of oxygen at tissue levels, and the resulting imbalance of acid and bases. As mediators inducing stress responses, the diverse endocrine systems including glucocorticoid hormones and the autonomic nervous system centered on sympathetic nerves are considered to be major factors. Recent studies also speculated the role of immune system including the release of cytokines and their interaction ${ }^{1,2}$.
Previous studies on cytokines secretion related to liver transplantation in patients with liver failure mostly focused on the types and subtypes of cytokines secreted during each stage of surgery ${ }^{3}$, the serial changes in their concentrations during the surgery ${ }^{4}$, and their relation to postoperative outcome after transplantation 5,6. Additionally, several studies to elucidate the following issues have been done; factors influencing the secretion of cytokines during liver transplantation 7,8 , pro-inflammatory and anti-inflammatory cytokine balance during liver transplantation surgery ${ }^{9}$, and the changes in the ratio of T helper 1 (Th 1) to T helper 2 (Th 2), and T helper 17 (Th 17) to regulatory $\mathrm{T}$ (Treg) cell signature 
cytokines during liver transplantation surgery with tools to change on the levels of Th 1, Th 2, Th17 and Treg cell signature cytokines ${ }^{10}$. In this review article, we aim to introduce these studies regarding cytokines secretion during liver transplantation and to suggest study directions for better outcomes of the patients.

\section{What Cytokines are Secreted during Each Stage of Liver Transplantation Surgery?}

Cytokines are substances secreted from several types of cells, including lymphocytes, monocytes, granulocytes, endothelial cells and synovial cells, which mediate immune and inflammatory reactions. TNF-alpha, IL-1, IL-2, IL-6, and IL-8 are proinflammatory cytokines that are secreted in response to stress reactions, such as sepsis and tissue damage, inducing hypotension and high fever as well as accelerating the production of acute phase proteins. The pro-inflammatory cytokines also enhance cell-mediated immunity and chemotaxis of inflammatory cells, and thus they play a role in protecting the human body from external stress ${ }^{11}$. In contrast, IL-1 receptor antagonist, IL-4, IL-10, IL-11, and IL-13 are anti-inflammatory cytokines that suppress excess inflammatory reactions and thus play a role in restricting the adverse influence they induce ${ }^{12}$.

Studies on cytokines related to liver transplantation have primarily addressed the fluctuation in the concentrations of cytokines during and following surgery. Boros et al ${ }^{5}$ found that TNF-alpha and IL-1 did not rise significantly prior to graft perfusion, and IL-6 and IL-8 substantially increased along time course of the surgery. Santiago et al ${ }^{3}$ reported more concrete findings that IL- 4 showed a peak value 20 min after reperfusion, IL-10 increased progressively, reaching a maximum $1 \mathrm{hr}$ after liver transplantation surgery, TNF-alpha reached maximum plasma level 20 min after reperfusion, and IL-6 was the first cytokines involved in the inflammatory response during transplantation surgery. Hassan et al ${ }^{6}$ reported study results that TNF-alpha and IL-4 had almost no changes in concentration during liver transplantation surgery, while IL-2 and IFN-gamma showed modest increase, and IL- 6 and IL- 1 showed huge increase in concentration. Overall, it is a common opinion that while TNF-alpha, IL-2, IL-4, and IFN-gamma have little or modest increase in concentration during liver transplantation surgery, IL-6 and IL-10 show high level of changes in concentration during surgery. Meanwhile, as a result of tracking the concentration of cytokines for 7 days after liver transplantation surgery, IL-6 and IL-10 which showed sharp increase in concentration during surgery restored to their original concentrations 2-3 days after surgery ${ }^{6}$.
Even though cytokines level represents the response of immune system due to stress, there are several cases where the results are indecisive. The reasons are as follows: 1) cytokines concentration in healthy state is basically very low so that it is difficult to sensitively detect the change in concentration, 2) even though cytokines are secreted in immune cells due to some stressor, the actual blood concentration measured may not be high due to the membrane structure that blocks their spread into blood stream, 3) since the half-life of cytokines is short in most cases (0-20 minutes), the concentration might fluctuate depending on the time of blood sampling.

\section{The Association of Cytokines Secretion with the Prognosis after Liver Transplantation}

There have been many evidences that the amount of cytokines secretion during liver transplantation is related with postoperative graft prognosis. The immune system secretes cytokines as a result of stress response. Therefore, it is reckonable that the well-being of a patient including graft survival can be influenced by the level of stress and the amount of cytokines secretion as a result. This is supported by study results on the relation between cytokines concentration and bilirubin concentration which is directly related to the function of graft liver. According to Hassan et al's study ${ }^{6}$, serum bilirubin concentration reached its highest level 1-6 hours after liver transplantation surgery, at the same time, IL-6 and IL-10 concentrations also reached their highest points. This means that the cytokines secretion was the highest when graft function was in its lowest point. They also tracked the concentration of IL-6 and total bilirubin concentration in liver transplant recipients with postoperative complication; bilirubin fell to almost normal values but not in cases of postoperative complications, where IL-6 showed values four times higher than those of liver transplant recipients who did not show postoperative complications. They concluded that IL-6 and IL-10 plasma concentrations and serum bilirubin level might be useful as a predictive factor for postoperative complications in liver transplantation. Other reports support the relationship between cytokines concentration and graft function. Boros et al ${ }^{5}$ stated that IL-1, IL-6 and IL-8 levels in first $20 \mathrm{ml}$ of hepatic effluent were higher in patients with poor early graft function. They also found that the levels of IL-6, IL-8 and TNF-alpha on postoperative day 1 were higher in patients with poor early graft function. Mueller et al ${ }^{13}$ also stated that various cytokines including IL-2 correlate with graft function, mentioning the mechanism that excessive secretion of cytokines 
promotes graft inflammation and results in a loss of graft function.

There are evidences that organ function other than that of liver is also related with cytokines concentration level after liver transplantation. Burke et al 14 found that following orthotopic liver transplantation, IL-6 and TNF-alpha fell to nearcontrol levels as renal function improved.

On the other hand, there are opinions that it is too much to predict the outcomes after liver transplantation through cytokines secretion. Maring et al 15 addressed that monitoring endotoxins and cytokines is of very limited value in predicting outcome, such as incidence of bacteremia, abdominal infection, and rejection.

\section{Factors Influencing the Concentration of Cytokines during Liver Transplantation}

There are various factors that influence the secretion of cytokines in end-stage liver failure patients in need of liver transplantation surgery, which include severity index of liver disease, amount of intraoperative transfusion and hemodynamic parameters during surgery $4,5,16-19$.

Above all, patients with end-stage liver failure show different aspects of cytokines secretion compared to normal healthy person. According to the study of Hong et al 7 , preoperative plasma concentrations of IL-6, TNF-alpha and IL-10 in patients with liver failure were significantly higher than those in healthy subjects (donors), which confirms the difference in the degree of stress responses between the two groups (Figure 1). It is the result of

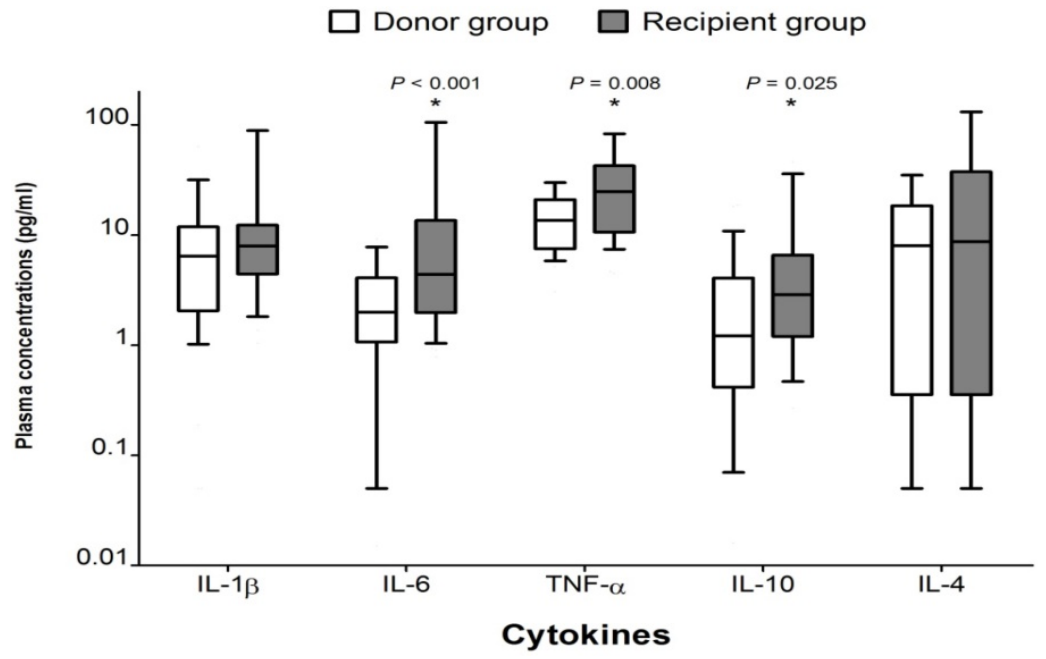

Figure 1. Comparison of preoperative cytokine concentrations between groups. The box plots show the median (line in the middle of the box), interquartile range (box), and 10th and 90th percentiles (whiskers). * $P<0.05$ versus donor group. [from Ref. 16. Hong SH, Kim JE, Cho ML, Heo YJ, Choi JH, Choi JH, Lee J. Comparison of the Child-Turcotte-Pugh classification and the model for end-stage liver disease score as predictors of the severity of the systemic inflammatory response in patients undergoing living-donor liver transplantation. J Korean Med Sci 2011; 26: 1333-1338.] dysfunction of multiple organs, including the liver, systemic inflammation and stress responses to them. So, by measuring the levels of plasma cytokines, we can indirectly determine the degree of systemic inflammation and stress responses in a patient with liver failure.

Studies on factors influencing cytokines secretion during liver transplantation surgery have been active. Faybik et al ${ }^{4}$ conducted a study regarding the influence of relief of splanchnic congestion by usage of VVP on the secretion of cytokines in anhepatic phase and reported that cross clamping contributes more to inflammatory cell activation than the use of VVP in anhepatic phase.

There are evidences that the amount of transfusion during liver transplantation surgery affects cytokines secretion. Lee et al ${ }^{16}$ stated that the amount of intraoperative transfusion was positively related with IL-6 concentration of postoperative day 7 $\left(\mathrm{r}^{2}=0.718, \mathrm{P}=0.013\right)$. As a result of tracking serial changes in the IL-6 concentration, Miki et al ${ }^{17}$ stated that patients whose operative blood transfusion requirement was 10 unit of packed red cell or more showed higher IL-1, IL-6 and TNF-alpha concentrations after graft reperfusion, and concluded that increased blood loss induced increased pro-inflammatory cytokines.

Intraoperative hemodynamic parameters such as systemic vascular resistance (SVR) also show relevance with cytokines concentration. Arranz et al 18 described in their study that there was positive correlation between the baseline values of IL-6 with the SVR, and proclaimed that the baseline values of IL-6 may be a prognostic factor for hemodynamic performance during liver transplantation surgery. Lee et al 19 reported similar results that there was a significant correlation between IL-6 levels and SVR in cirrhosis. They concluded that IL-6 plays a significant role to induce vasodilatation. Additionally, they found that TNF-alpha induces NO, resulting decrease in SVR. It is an interesting fact that decrease in SVR, which is a distinctive hemodynamic characteristic in patients with end-stage liver disease, is related to cytokines concentration.

There have been studies on the difference in cytokines secretion depending on the underlying disease that caused hepatic failure. According to a study conducted by Koh et al 8 , there was no difference in the concentration of cytokines based on the underlying 
diseases (viral hepatitis vs. alcoholic hepatitis) that induce liver failure (Figure 2). They concluded that regardless of the disease that caused liver failure, the differences in diseases did not influence the concentrations of cytokines. This implies that the induction of the inflammatory and stress reactions which occurs with viral or alcoholic hepatitis is not different.

On the other hand, the factors that actually affect the concentrations of cytokines involved the model for end-stage liver disease (MELD) score ${ }^{8}$ (Figure 3), which is a reliable index associated with the severity and prognosis of liver failure patients. The MELD score is calculated by applying values to the variables in the following formula: (MELD score $=9.57 \mathrm{x}$ $\log _{\mathrm{e}}[$ creatinine $\mathrm{mg} / \mathrm{dL}]+3.78 \times \log _{\mathrm{e}}[$ bilirubin $\mathrm{mg} / \mathrm{dL}$ ] $\left.+11.20 \times \log _{\mathrm{e}}[\mathrm{INR}]+0.643\right)^{20}$. This corresponds with the study results of Boros et al ${ }^{5}$ that disease severity in terms of UNOS classification was associated with preoperative concentrations of IL-6 and IL-8. They also found out that secretion of IL-6 and IL-8 increased by 2-4 times in Child-Turcotte-Pugh (CTP) classification $\mathrm{C}$ group compared to $\mathrm{B}$ group.

There were studies on the concrete factors that influence the severity of systemic inflammatory reaction in liver failure patients. Cazzaniga et al 21 demonstrated that the prevalence and in hospital outcome of systemic inflammatory response in patients with cirrhosis were correlated with high serum bilirubin level, high PT-INR, and high serum creatinine level, all of which are constituent factors for MELD scores. Also, mediators of systemic inflammatory response may exacerbate the neuropsychological effects of hyperammonemia in cirrhosis 22. Additionally, bacterial translocation, the main bacterial entry route into the cirrhotic host, significantly increased in CTP classification C patients (31\%), compared with CTP B (8\%) and CTP A (3\%) patients ${ }^{23}$. Thus, we can conclude that the systemic inflammatory response that occurs in end-stage liver disease patients result in a more severe level of preoperative CTP classification and MELD score.
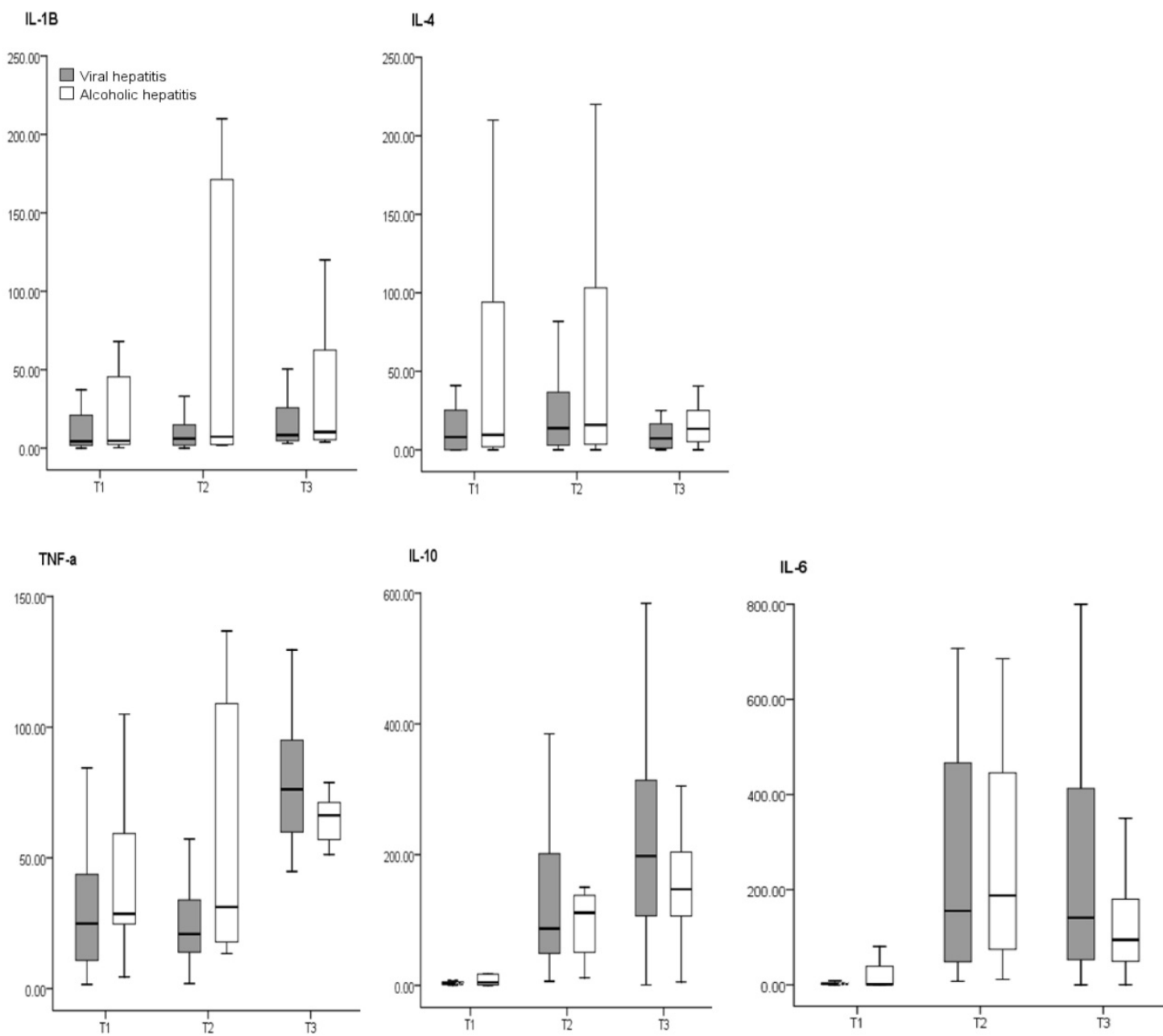

Figure 2. Comparison of cytokines concentrations between disease groups. The box plots show the median (thick line in the box), interquartile range (box), and range (whiskers). All cytokines concentrations are $\mathrm{pg} / \mathrm{ml}$ in value. T1: after induction of anesthesia, T2: $60 \mathrm{~min}$ after the start of anhepatic stage, T3: $60 \mathrm{~min}$ after reperfusion. There are no differences between disease groups. [from Ref. 17. Koh H], Ryu KH, Cho ML, Heo Y], Lee J. Factors influencing the concentration of cytokines during liver transplantation. Transplant Proc 2010; 42: 3617-3619.] 

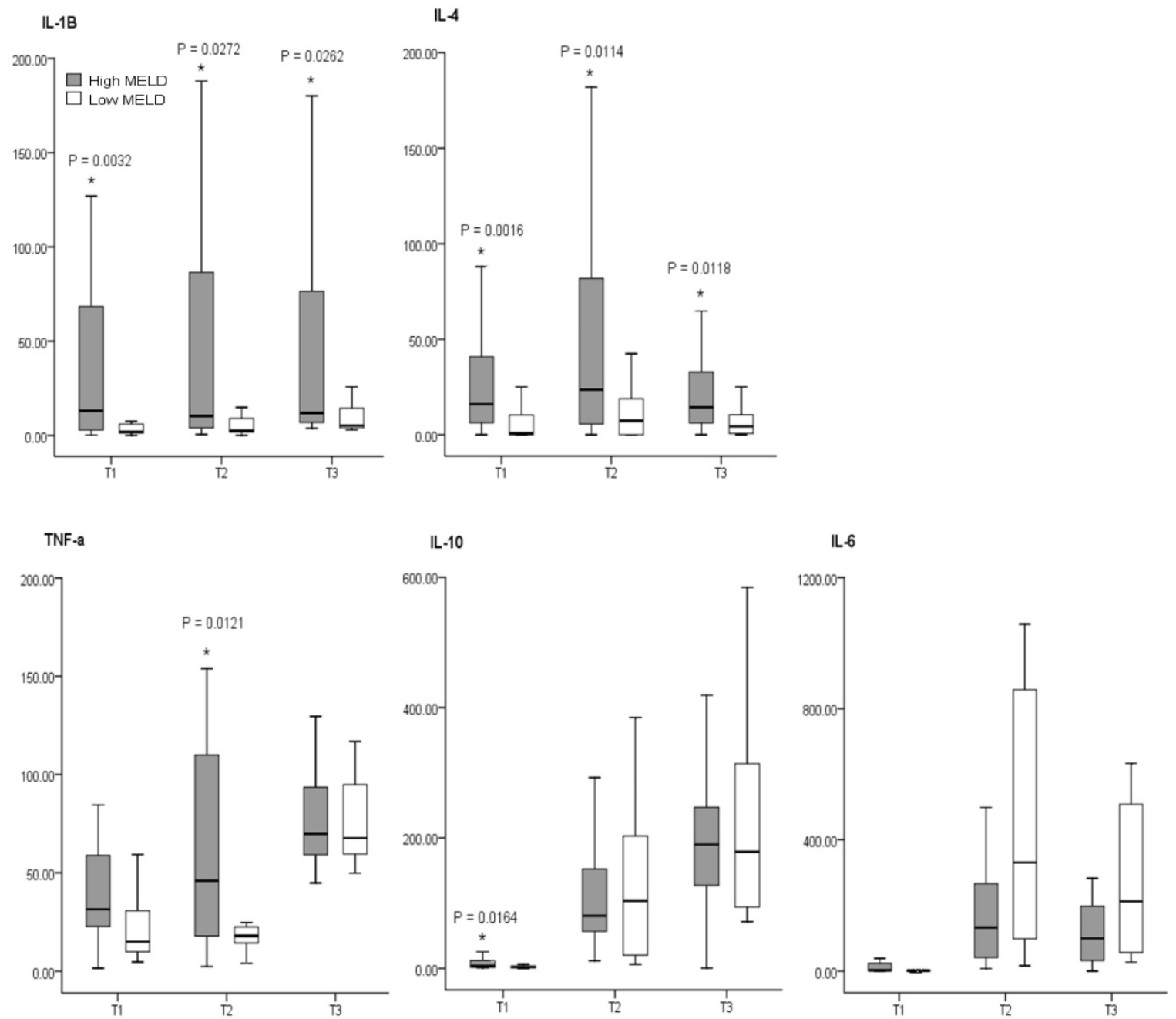

Figure 3. Comparison of cytokines concentrations between MELD-based groups. The box plots show the median (thick line in the box), interquartile range (box), and range (whiskers). All cytokines concentrations are $\mathrm{pg} / \mathrm{ml}$ in value. MEDL: model for end-stage liver disease, High MELD 220 , Low MELD<20, Time 1: after induction of anesthesia, Time 2: $60 \mathrm{~min}$ after the start of anhepatic stage, Time 3: $60 \mathrm{~min}$ after reperfusion. *: P<0.05 vs Low MELD. [from Ref. 17. Koh HJ, Ryu KH, Cho ML, Heo YJ, Lee J. Factors influencing the concentration of cytokines during liver transplantation. Transplant Proc 2010; 42: 3617-3619.]

Severity index of liver disease, however, does not influence the secretion of cytokines throughout the whole process of liver transplantation surgery. According to study results by Hong et al 7 , though the presurgical CTP classification and MELD score showed a close relationship with the level of cytokines at preoperative stage, that relationship tended to diminish or disappear as the liver transplantation surgery progressed. The main factor determining cytokines secretion during liver transplantation surgery is not the presurgical CTP classification or MELD score, but the surgical stress from extensive tissue injury, deterioration of acid-base balance, massive bleeding and transfusion, and severe hemodynamic instability. These points are supported by the research by Miki et al 17 that the larger the transfusion volume during surgery, the more accelerated the secretion of pro-inflammatory cytokines and thus higher the concentrations of IL-1beta and IL-6 during surgery. In addition, by the research results reported by Lee et al ${ }^{16}$, the larger the transfusion volume during surgery, the higher the concentration of IL-6 on days 1 and 7 after surgery.

As we have reviewed, there are various factors that influence the secretion of cytokines during liver transplantation surgery. However, one should keep in mind that studies on the factors influencing cytokines secretion during liver transplantation surgery sometimes may not be simply done. The reasons are as follows: 1) it might be hard to evaluate the pure influence of stressors on cytokines secretion since the secretion of cytokines is also affected by other non-specific causes such as various anesthetic agents and anesthetic techniques, 2) although cytokines are secreted in immune cells due to some stressor, the influence of that stressor can be masked due to membrane structure blocking its spread to blood stream, resulting in low blood concentration when actually measured, 3 ) the reaction modality might not be distinctive since the pro-inflammatory cytokines 
secreted are offset by anti-inflammatory cytokines, thus maintaining hemostasis. Therefore, when conducting a study on the factors influencing the secretion of cytokines, it is important to equalize other conditions, such as anesthetic agents and anesthetic techniques, that influence the secretion of cytokines, as well as to measure the concentration of both proand anti-inflammatory cytokines to understand the secretion modality.

\section{Pro-inflammatory and Anti-inflammatory Cytokine Balance during Liver Transplantation Surgery}

In healthy condition, a delicate equilibrium between pro- and anti-inflammatory cytokines, and thus homeostasis is maintained. However, in stress state as trauma, sepsis, and neoplasms, such homeostasis is disrupted by excess elevation of proinflammatory cytokines, which ultimately increase the morbidity and mortality rate ${ }^{24}$. When excess pro-inflammatory cytokines are released during stress, anti-inflammatory cytokines are subsequently secreted to restore the balance. This process is called the phase of early pro-inflammatory and late anti-inflammatory cytokines $25-27$.

Koh et al ${ }^{9}$ performed a study regarding this issue and demonstrated this condition. Their results showed that the cytokines released markedly in patients with cirrhotic hepatitis during liver transplant surgery include IL-6 (a pro-inflammatory cytokine whose concentration increased as much as 40 times compared to its preoperative values at the anhepatic stage) and IL-10 (an anti-inflammatory cytokine whose concentration was 60 times higher than its preoperative value at the neohepatic phase) (Table 1). They concluded that interleukin-6 and interleukin-10 are early pro- and late anti-inflammatory cytokines, respectively, and play a major role in cytokine balance during live-donor liver transplant surgery.

Table 1. Time Couse of Cytokines Concentrations in Recipient Group.

\begin{tabular}{lllll}
\hline & Time 1 & Time 2 & Time 3 & P-Value \\
\hline IL-1 $\beta$ & $8.42(4.5,12.3)$ & $9.72(4.0,33.7)$ & $7.86(3.1,41.3)$ & 0.956 \\
IL-6 & $4.48(2.1,11.1)$ & $176.35(51.0$, & $135.66(52.1,327.3)$ & $<0.001$ \\
& & $458.9)$ & & \\
TNF- a & $26.02(11.2,47.8)$ & $36.14(15.4,65.3)$ & $54.65(21.3,67.3)$ & 0.002 \\
IL-10 & $2.98(1.2,5.7)$ & $95.32(48.6,198.2)$ & $184.35(96.7,298.8)$ & $<0.001$ \\
IL-4 & $8.16(0.5,27.7)$ & $13.85(3.2,40.8)$ & $7.83(1.1,21.4)$ & 0.687 \\
\hline
\end{tabular}

Values are median with quartile $(25 \%, 75 \%)$. All cytokines concentrations are $\mathrm{pg} / \mathrm{ml}$ in value. Time 1: after induction of anesthesia, Time 2: $60 \mathrm{~min}$ after the start of anhepatic stage, Time 3: $60 \mathrm{~min}$ after reperfusion.

[from Ref. 26. Koh HJ, Joo J, Cho ML, Her YM, Hwang JE, Lee J. Pro-inflammatory and anti-inflammatory cytokine balance in patients with cirrhotic hepatitis during live-donor liver transplant. Exp Clin Transplant 2013; 11: 39-43.]
IL-6 and IL-10 are pleiotropic, and they have both positive and negative sides on the clinical outcome. For IL-6, in addition to its original pro-inflammatory effect, it also has broad hepatoprotective effects ${ }^{28,29}$. For IL-10, even though it can reduce the magnitude of the inflammatory response and improve outcomes 26,30,31, it also can exacerbate T-cell dysfunction, decrease T-cell apoptosis, reduce antimicrobial function, and increase mortality in bacterial models of sepsis ${ }^{32}$. Therefore, the consequence of the increased concentration of IL-6 and IL-10 during surgery should not be limited to regulation of the magnitude of the pro- and anti-inflammatory response. The clinical implication of IL-6 and IL-10 in maintaining cytokine balance during liver transplant surgery must be elucidated in future studies.

\section{T Helper 1 and T Helper 2, and T Helper 17 and regulatory $T$ Cell Signature Cytokines Secretion during Liver Transplantation Surgery}

$\mathrm{T}$ helper cells are initially capable of production of many cytokines and are prompted into a more restricted and focused pattern of cytokine production depending on signals received at stress state. The production of a specific type of $\mathrm{T}$ helper cell is determined by differentiation of precursor helper $\mathrm{T}$ cells (Th 0 ) into Th 1 or Th 2 cells. Th 1 cells produce IFN-gamma and TNF-alpha, and favor cell-mediated immune responses. Th 2 cells produce IL- 4 and IL-6, and favor humoral immunity in terms of control of antibody production ${ }^{33}$. It is known that polarization of $\mathrm{T}$ helper cells toward either a Th 1 or Th 2 can significantly influence subsequent immune responses and provoke postoperative complications 34. Therefore, understanding the features of immunomodulation during liver transplantation surgery is important for improving patient outcomes.

In fact, several researchers have elucidated the effect of shifting of the Th $1 /$ Th 2 ratio on clinical outcomes. Tan et al 35 showed that probiotics could shift the Th 1/Th 2 (IFN-gamma/IL-4) ratio toward Th 1 in severe traumatic brain-injured patients who had a decreased Th 1/Th 2 ratio, and could result in decreased nosocomial infection rates. Decker et al 36 found that surgery itself decreased the Th $1 /$ Th 2 ratio, and claimed that the decreased Th $1 /$ Th 2 ratio resulted in suppressed cell-mediated immunity after surgery. On the contrary, Inada et al ${ }^{37}$ reported that dexmedetomidine showed antitumor immunity effect by decreasing Th 1/Th 2 ratio. Thus, surgeons and anesthesiologists should not ignore the potential influence of immunomodulation during major 
surgical procedures on the perioperative outcomes of immunity.

One interesting study to actively modulate $\mathrm{T}$ helper subset cell signature cytokine was done by Chung et al 10. They investigated the effects of intravenous magnesium pretreatment on levels of $\mathrm{T}$ helper cytokines and their ratio at allograft reperfusion in patients undergoing living donor liver transplantation. When magnesium was administered just before the reperfusion of allografts, the post-reperfusion concentration of IFN-gamma, a Th 1 subset cell signature cytokine, was significantly lower than that in the control group. On the other hand, the post-reperfusion concentration of IL-4 and IL-6, which are Th 2 subset cell signature cytokines, were significantly higher in the magnesium group than in the control group (Figure 4). This suggests that magnesium pretreatment shifted the Th 1-to-Th 2 balance towards Th 2 and thus plays an immunomodulatory role after allograft reperfusion.

The mechanism of magnesium pretreatment on immunomodulation is associated with its serum level. Hypomagnesemia, one of the main manifestations in patients with hepatic failure, is known to increase C-reactive protein and pro-inflammatory cytokines such as TNF-alpha, which can stimulate free radical production in endothelial cells and circulating white blood cells ${ }^{38}$. Conversely, normomagnesemia exhibits a protective role against harmful inflammatory reactions 39,40 . The correction of hypomagnesemia before reperfusion in their study may have resulted in the decreased secretion of pro-inflammatory cytokines by Th 1 lymphocytes and the increased production of anti-inflammatory cytokines by Th 2 lymphocytes, shifting Th 1/Th 2 balance toward Th 2 and minimizing harmful inflammatory reactions.

(A) Th1 cytokines
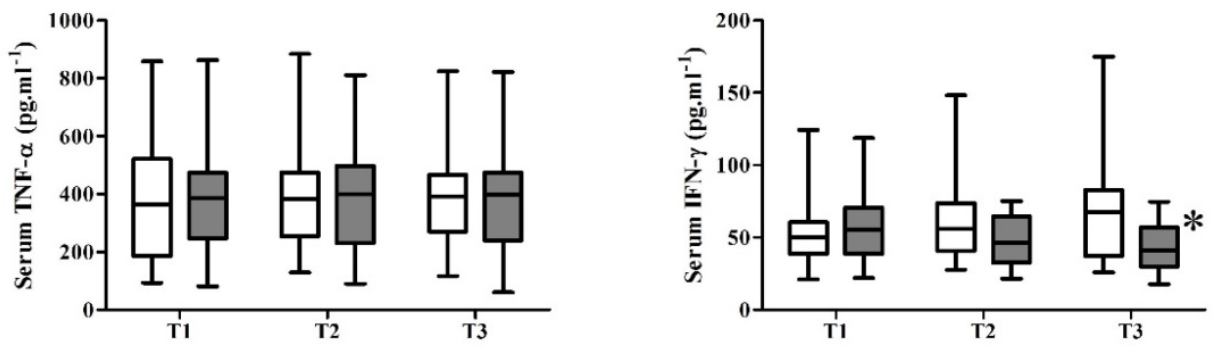

(B) Th2 cytokines
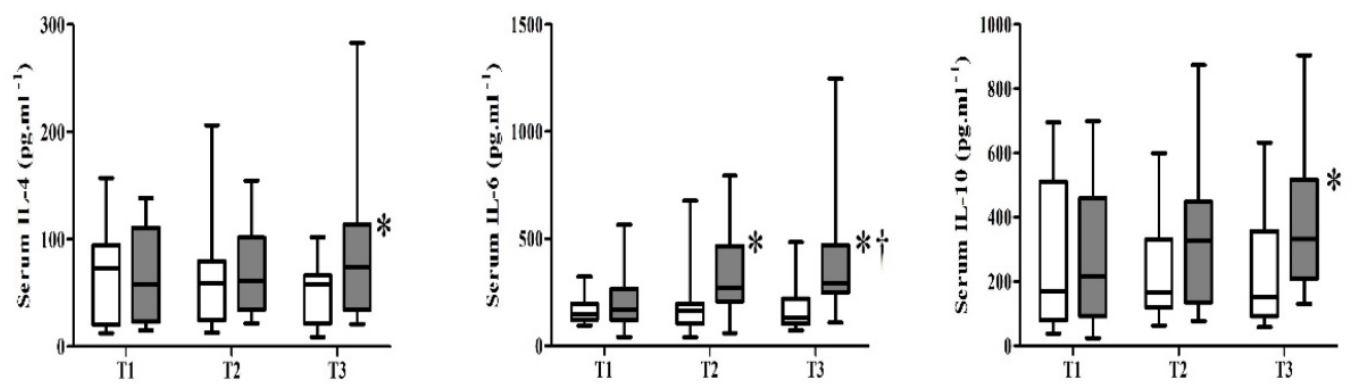

(C) Ratio of the Th1/Th2 cytokines
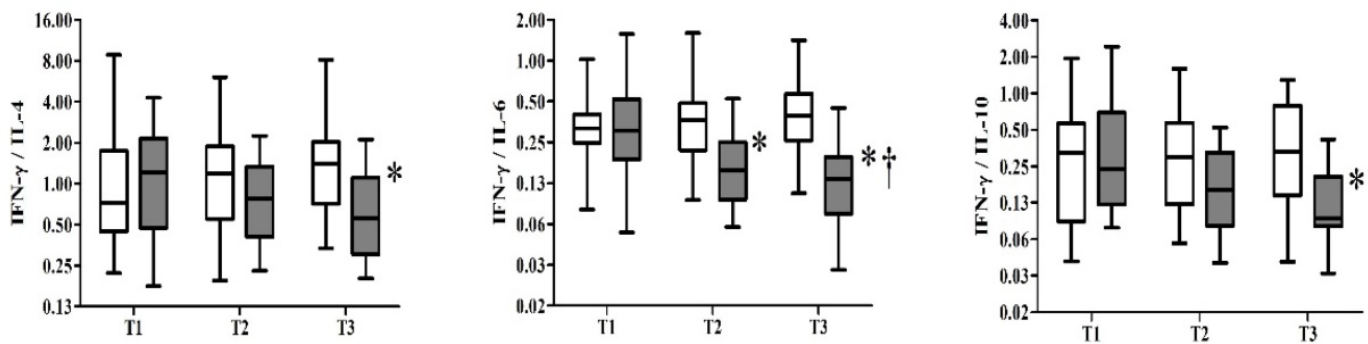

Figure 4. Comparison of the serum concentrations of cytokines in patients receiving magnesium ( $\square$ ) or saline ( $\square$ ). (A) Th 1 cytokines; (B) Th 2 cytokines; (C) the ratio of Th 1 to Th 2 cytokines. The box plot shows medians and quartiles. Error bars represent the $90^{\text {th }}$ and $10^{\text {th }}$ percentiles. *p $<0.05$ between the groups at the time point indicated; $\nmid p<0.05$ between the two groups over time. T1, before infusion of the study solution at 20 min before the start of the neohepatic phase. T2, 5 min after reperfusion. T3, 30 min after reperfusion. [from Ref. 37. Chung HS, Park CS, Hong SH, Lee S, Cho ML, Her YM, Sa G], Lee J, Choi JH. Effects of magnesium pretreatment on the levels of $\mathrm{T}$ helper cytokines and on the severity of reperfusion syndrome in patients undergoing living donor liver transplantation. Magnes Res 2013; 26: 46-55.] 
Recent studies have shown that the roles of Th1, Th2, Th17, and Treg are important for graft survival after liver transplantation. Zhou et al. ${ }^{41}$ reported that imbalance between Th 17 and Treg cells in the rodent liver graft model was involved in allograft rejection. In detail, Th 17 cells and related cytokines (IL-17) were increased, whereas Treg cells and related cytokines (IL-10) were lower in the allograft group compared with isograft and control group. In addition, they found that neutralization of IL-17 in allograft group prolonged the survival, improved liver function, and increased Treg cells in the liver. $\mathrm{Li}$ et al. ${ }^{42}$ have reported similar results in rat liver transplantation, which shows that Th 17/Treg imbalance plays a potential role in the pathogenesis of acute transplant rejection. On the other hand, Yang et al. ${ }^{43}$ have identified the complex effects of Th 17, Treg, Th 1, and Th 2 on liver graft rejection. According to their study, bone marrow mesenchymal stem cells injected into mice with orthotopic liver transplantation relieved acute rejection and increased survival rate. The mechanism is that bone marrow mesenchymal stem cells increase Th 2 and T reg cells, and decrease Th 1 and Th 17 cells. Taken together, we can conclude that Treg and Th 2 work together to positively influence the mitigation of acute rejection and the increase in graft survival rate.

\section{Future Study Direction}

Future study direction should focus on utilizing cytokines in order to improve clinical outcomes including enhancement of graft survival. Among several different lymphoid cell types which are known to demonstrate immune regulatory function, a subset of CD4 T cells or Treg is the most important. Strategies to prevent liver transplant rejection whilst minimizing long-term immunosuppression are currently under intense investigation with regulatory T cells nearing clinical application. Tregs control acute and delayed allograft rejection ${ }^{44}$. Immune tolerance of Treg is demonstrated by expressing high affinity IL-2 receptor alpha chain 45 , meaning that the effect of Treg is realized by the action on cytokines. According to a recent study, the Treg cell therapy to induce tolerance in liver transplant recipients is successful ${ }^{46}$. With the current viewpoint, however, there are following concerns: 1) Treg therapy is not effective in immune competent recipients, 2) it is difficult to obtain sufficient number, purity and stability of Treg, 3) there can be an increase in the possibility of infection and malignancy of recipients due to generalized suppression of adoptive immune system. If these concerns are resolved, the Treg would be a great tool that can be used to reduce graft rejection after transplantation.

\section{Conclusions}

Liver transplantation is a major surgical procedure with various kinds of stressors including massive transfusion, clamping of major vessels, acid-base imbalance and ischemia-reperfusion injury; together with liver failure, it acts as a stressor threatening the homeostasis of the body. Therefore, the secretion of cytokines is very active and dramatic during liver transplantation surgery as a response to stress.

We reviewed the previous literature regarding the release of cytokines and their interactions in liver transplantation surgery. In detail, this review describes the type of the cytokines released in each surgical stage, certain cytokines as a factor for postoperative prognosis, various factors affecting the release of cytokines, and the balance of pro- and anti-inflammatory cytokines, Th1 and Th2, and Th 17 and Treg signature cytokines during liver transplantation.

Though we proposed various factors that influence the secretion of cytokines during liver transplantation surgery, we do not think that it is enough. Other factors that influence the secretion of cytokines must be figured out since it is closely related to clinical outcomes. Also, how the secretion of cytokines affect clinical outcomes should be elucidated in detail henceforth. When tools to artificially manipulate the cytokines are found and applied in clinical situations, we believe that prognosis after liver transplantation surgery will improve significantly.

\section{Competing Interests}

The authors have declared that no competing interest exists.

\section{References}

1. Barker CE, Ali S, O'Boyle G, Kirby JA. Transplantation and inflammation: implications for the modification of chemokine function. Immunology. 2014; 143: 138-45.

2. Sato Y, Tomiyama C, Yamamoto S, Oya H, Kobayashi T, Kokai H, et al. Perioperative immunological differentiation in liver cirrhotic patients who underwent living related liver transplantation. Hepatogastroenterology. 2013; 60: 666-8.

3. Santiago F, Bueno P, Olmedo C, Comino, Hassan L, Ferrón-Celma I, et al. Time course of intraoperative cytokine levels in liver transplant recipients. Transplant Proc. 2006; 38: 2492-4.

4. Faybik $\mathrm{P}$, Hetz $\mathrm{H}$, Krenn $\mathrm{CG}$, Baker A, Berlakovich GA Steltzer $\mathrm{H}$. Perioperative cytokines during orthotopic liver transplantation without venovenous bypass. Transplant Proc. 2003; 35: 3019-21.

5. Boros P, Suehiro T, Curtiss S, Sheiner P, Emre S, Guy S, et al. Differential contribution of graft and recipient to perioperative TNF- $\alpha$, IL-1 $\beta$, IL-6 and IL-8 levels and correlation with early graft function in clinical liver transplantation. Clin Transplant. 1997; 11: 588-92.

6. Hassan L, Bueno I, Ferrón-Celma I, Ramia JM, Garrote D, Muffak K, et al. Early postoperative response of cytokines in liver transplant recipients. Transplant Proc. 2006; 38: 2488-91.

7. Hong $\mathrm{SH}, \mathrm{Kim}$ JE, Cho ML, Heo JY, Choi JH, Choi JH, et al. Comparison of the Child-Turcotte-Pugh classification and the model for end-stage liver disease score as predictors of the severity of the systemic inflammatory response in patients undergoing living-donor liver transplantation. J Korean Med Sci. 2011; 26: 1333-8. 
8. Koh HJ, Ryu KH, Cho ML, Heo YJ, Lee J. Factors influencing the concentration of cytokines during liver transplantation. Transplant Proc. 2010; 42: 3617-9.

9. Koh HJ, Joo J, Cho ML, Her YM, Hwang JE, Lee J. Pro-inflammatory and anti-inflammatory cytokine balance in patients with cirrhotic hepatitis during live-donor liver transplant. Exp Clin Transplant. 2013; 11: 39-43.

10. Chung HS, Park CS, Hong SH, Lee S, Cho ML, Her YM, et al. Effects of magnesium pretreatment on the levels of $\mathrm{T}$ helper cytokines and on the severity of reperfusion syndrome in patients undergoing living donor liver transplantation. Magnes Res. 2013; 26: 46-55

11. Ng CS, Lau KK. Surgical trauma and immune functional changes following major lung resection. Indian J Surg. 2015; 77: 49-54.

12. Briem-Richter A, Leuschner A, Haag F, Grabhorn E, Ganschow R. Cytokine concentrations and regulatory $\mathrm{T}$ cells in living donor and deceased donor liver transplant recipients. Pediatr Transplant. 2013; 17: 185-90.

13. Mueller AR, Platz KP, Haak M, Undi H, Müller C, Köttgen E, et al. The release of cytokines, adhesion molecules, and extracellular matrix parameters during and after reperfusion in human liver transplantation. Transplantation. 1996; 62: 1118-26.

14. Burke GW, Cirocco R, Roth D, Fernandez J, Allouche M, Markou M, et al. Activated cytokine pattern in hepatorenal syndrome: fall in levels after successful orthotopic liver transplantation. Transplant Proc. 1993; 25: 1876-7.

15. Maring JK, Klompmaker IJ, Zwaveling JH, van Der Meer J, Limburg PC, Slooff MJ. Endotoxins and cytokines during liver transplantation: changes in plasma levels and effects on clinical outcome. Liver Transpl. 2000; 6: 480-8.

16. Lee KW, Son TS, Joh JW, Kim SJ, Park JH, Chon SE, et al. Perioperative cytokine response after liver transplantation. Transplant Proc. 2003; 35: 407-8.

17. Miki C, Iriyama K, Gunson BK, Mayer AD, Buckels JA, McMaster P. Influence of intraoperative blood loss on plasma levels of cytokines and endotoxin and subsequent graft liver function. Arch Surg. 1997; 132: 136-41.

18. Arranz J, Soriano A, Garcia I, García I, Concepción MT, Navarro J, et al. Effect of proinflammatory cytokines (IL-6, TNF- $\alpha$, IL-1 $\beta$ ) on hemodynamic performance during orthotopic liver transplantation. Transplant Proc. 2003; 35: $1884-7$

19. Lee FY, Lu RH, Tsai YT, Lin HC, Hou MC, Li CP, et al. Plasma interleukin-6 levels in patients with cirrhosis. Relationship to endotoxemia, tumor necrosis factor-alpha, and hyperdynamic circulation. Scand J Gastroenterol. 1996; 31: 500-5.

20. Flodén A, Castedal M, Friman S, Olausson M, Backman L. Calculation and comparison of the model for end-stage liver disease (MELD) score in patients accepted for liver transplantation in 1999 and 2004. Transplant Proc. 2007; 39: $385-6$

21. Cazzaniga M, Dionigi E, Gobbo G, Fiorette A, Monti V, Salerno F. The systemic inflammatory response syndrome in cirrhotic patients: relationship with their in-hospital outcome. J Hepatol. 2009; 51: 475-82.

22. Shawcross DL, Davies NA, Williams R, Jalan R. Systemic inflammatory response exacerbates the neuropsychological effects of induced hyperammonemia in cirrhosis. J Hepatol. 2004; 40: 247-54.

23. Cirera I, Bauer TM, Navasa M, Vila J, Grande L, Taurá P, et al. Bacterial translocation of enteric organisms in patients with cirrhosis. J Hepatol. 2001; 34: $32-7$

24. Elenkov IJ, Chrousos GP. Stress hormones, proinflammatory and anti-inflammatory cytokines, and autoimmunity. Ann N Y Acad Sci. 2002; 966: 290-303

25. Kimura F, Shimizu H, Yoshidome H, Ohtsuka M, Miyazaki M. Immunosuppression following surgical and traumatic injury. Surg Today. 2010; 40: 793-808.

26. Lyn-Kew K, Standiford TJ. Immunosuppression in sepsis. Curr Pharm Des. 2008; 14: 1870-81.

27. Elenkov IJ, Iezzoni DG, Daly A, Harris AG, Chrousos GP. Cytokine dysregulation, inflammation and well-being. Neuroimmunomodulation. 2005; 12: 255-69.

28. Fujiyoshi M, Ozaki M. Molecular mechanisms of liver regeneration and protection for treatment of liver dysfunction and diseases. J Hepatobiliary Pancreat Sci. 2011; 18: 13-22.

29. Hong $F$, Kim $W H$, Tian $Z$, Jaruga $B$, Ishac $E$, Shen $X$ et al Elevated interleukin-6 during ethanol consumption acts as a potential endogenous protective cytokine against ethanol-induced apoptosis in the liver: involvement of induction of Bcl-2 and Bcl-x(L) proteins. Oncogene. 2002; 21: $32-43$.

30. Scumpia PO, Moldawer LL. Biology of interleukin-10 and its regulatory roles in sepsis syndromes. Crit Care Med. 2005; 33 (Suppl 12): S468-S471.

31. Rodell CB, Rai R, Faubel S, Burdick JA, Soranno DE. Local immunotherapy via delivery of interleukin-10 and transforming growth factor $\beta$ antagonist for treatment of chronic kidney disease. J Control Release. 2015; 206: 131-9.

32. Oberholzer A, Oberholzer C, Moldawer LL. Interleukin-10: a complex role in the pathogenesis of sepsis syndromes and its potential as an anti-inflammatory drug. Crit Care Med. 2002; 30 (Suppl 1): S58-S63.

33. Kurosawa S, Kato M. Anesthetics, immune cells, and immune responses. J Anesth. 2008; 22: 263-77.

34. Cardinale F, Chinellato I, Caimmi S, Peroni DG, Franceschini F, Miraglia Del Giudice $\mathrm{M}$, et al. Perioperative period: immunological modifications. Int J Immunopathol Pharmacol. 2011; 24 (Suppl 3): S3-S12.

35. Tan M, Zhu JC, Du J, Zhang LM, Yin HH. Effects of probiotics on serum levels of Th1/Th2 cytokine and clinical outcomes in severe traumatic brain-injured patients: a prospective randomized pilot study. Crit Care. 2011; 15: R290.
36. Decker D, Schöndorf M, Bidlingmaier F, Hirner A, von Ruecker AA. Surgical stress induces a shift in the type-1/type-2 T-helper cell balance, suggesting downregulation of cell-mediated and up-regulation of antibody-mediated mmunity commensurate to the trauma. Surgery. 1996; 119: 316-25.

37. Inada T, Shirane A, Hamano N, Yamada M, Kambara T, Shingu K. Effect of subhypnotic doses of dexmedetomidine on antitumor immunity in mice. Immunopharmacol Immunotoxicol. 2005; 27: 357-69.

38. Malpuech-Brugère C, Nowacki W, Daveau M, Gueux E, Linard C, Rock E, et al. Inflammatory response following acute magnesium deficiency in the rat. Biochim Biophys Acta. 2000; 1501: 91-8.

39. Guerrero-Romero F, Bermudez-Peña C, Rodríguez-Morán M. Severe hypomagnesemia and low-grade inflammation in metabolic syndrome. Magnes Res. 2011; 24: 45-53.

40. Guerrero-Romero F, Rodríguez-Morán M. Hypomagnesemia, oxidative stress, inflammation, and metabolic syndrome. Diabetes Metab Res Rev. 2006; 22: 471-6.

41. Zhou Y, Yang X, Zhang H, Jiang J. The role of T helper type 17 /regulatory $\mathrm{T}$ cells in acute rejection after liver transplantation in rats. Transplantation 2015; 99: 1126-31.

42. Li J, Lai X, Liao W, He Y, Liu Y, Gong J. The dynamic changes of Th17/Treg cytokines in rat liver transplant rejection and tolerance. Int Immunopharmacol 2011; 11: 962-7.

43. Yang Y, Shen ZY, Wu B, Yin ML, Zhang BY, Song HL. Mesenchymal stem cells improve the outcomes of liver recipients via regulating CD4+ $\mathrm{T}$ helper cytokines in rats. Hepatobiliary Pancreat Dis Int 2016; 15: 257-65.

44. Wood KJ. Regulatory T cells in transplantation. Transplant Proc. 2011; 43: 2135-6.

45. Wood KJ, Bushell A, Hester J. Regulatory immune cells in transplantation. Nat Rev Immunol. 2012; 12: 417-30.

46. Safinia N, Vaikunthanathan T, Fraser H, Thirkell S, Lowe K, Blakemore L, et al. Successful expansion of functional and stable regulatory $\mathrm{T}$ cells for immunotherapy in liver transplantation. Oncotarget 2016; 7: 7563-77. 